\title{
Gesture based and Voice Enabled Device Switching of Basic Needs for Paralyzed Persons
}

\author{
Suneetha K. B*, Chaithra T. S, Harshitha K. S, Yashaswini H. J, Nithaksha N \\ Department of Computer Science and Engineering, Vidya Vikas Institute of Engineering and \\ Technology Mysuru, India
}

DOI: https://doi.org/10.21467/proceedings.1.69

Corresponding Author email: suneekb7043@gmail.com

\begin{abstract}
Due to spinal cord injury except head part human can lose controller over all his body parts. In such cases a helper is required for the patient at every time. For example if the patient requires fan then he should inform to his helper like this are many instances where he requires helper like in switching fan, lights, TV.. Etc. These purposes can be served without the need of another human help. In this project we can serve the purpose of controlling the home appliances without the need of a helper. Here we use accelerometer sensors which are attached to a head mask of the patient. Just only by the movement of the head the patient can control the home appliances on his own.
\end{abstract}

Index Terms-assistive technology, head gesture, human-robot interaction, inertial measurement unit, usability study

\section{INTRODUCTION}

For each and every person Communication is an essential part of human life. If communication is disturbed or impossible, the consequences are loneliness and isolation. It is well known that speech plays a key role in communication and it explains why humans also want to have speech as a means of communication/interaction with computers. Although human-like speech dialogue with computers is still far off, even with current state-of-the-art technology, the benefits and potential of speech processing are obvious. People make use of different languages to express their thoughts in a different way. But, it is difficult for the people who are affected, partially, by paralysis and stroke. Hence, there is a need to develop a platform for those people who are physically challengeable. An Embedded device shall address the above said problems. Paralysis is a life-changing condition, especially if the injury prohibits the person from going about the daily routines or jobs the way got used to doing it. Our goal is to provide these people an affordable solution that can make them independent for most of their day and enable them to work, create, and feel useful in the society. This project provides the controlling of home appliances, giving emergency alert with the head movement. An accelerometer will be placed on the center, topmost, position of the head an individual who is physically disabled or cannot speak due to paralysis. Any movement of the head in particular

This is an open access article under Creative Commons Attribution-NonCommercial 4.0 International (CC BY-NC 4.0) 
direction would cause the accelerometer to generate an output voltage. These output voltages corresponds to the change in gravity. These output voltages are fed as input to the microcontroller four commands are provided for controlling above parameters.

\section{LITERATURE SURVEY}

Hikaru Amano, Hidekuni Ogawa, Hiromichi Maki, Sosuke Tsukamoto, Yoshiharu Yonezawa, W. Morton Caldwell, "A remote drip infusion monitoring system employing Bluetooth" EMBC, IEEE. ISSN:1557-170X. We have developed a remote drip infusion monitoring system for use in hospitals. The system consists of several infusion monitoring devices and a central monitor. The infusion monitoring device employing a Bluetooth module can detect the drip infusion rate and an empty infusion solution bag, and then these data are sent to the central monitor placed at the nurses' station via the Bluetooth. The central monitor receives the data from several infusion monitoring devices and then displays graphically them. Therefore, the developed system can monitor intensively the drip infusion situation of the several patients at the nurses' station. Priyadharshini.R, Mithuna.S, VasanthKumar.U, KalpanaDevi.S, Dr.SuthanthiraVanitha.N, "Automatic Intravenous Fluid Level Indication System for Hospitals", IJRASET.ISSN: 2321-9653 During recent years due to the technological advancements many sophisticated techniques has been evolved for assuring fast recovery of the patients in hospitals. For good patient care in hospitals, assessment and management of patient's fluid and electrolyte need is the most fundamental thing required. All most in all hospital, an assist/nurse is responsible for monitoring the IV fluid level continuously. But unfortunately during most of the time, the observer may forget to change the saline bottle at correct time due to their busy schedule. This may leads to several problems to the patients such as backflow of blood, blood loss etc. To overcome this critical situation, a low cost RF based automatic alerting and indicating device is proposed where IR sensor is used as a level sensor. It is based on the principle that the IR sensor output voltage level changes when intravenous fluid level is below certain limit. A comparator is used to continuously compare the IR output with predefined threshold. When the transceiver output is negative then the Arduino controller identifies the fluid level is too low and it alerts the observer by buzzer and LCD at the control room indicates the room number of the patient for quick recovery. It describe that the approaches present can be mainly divided into "DataGlove based" and "Vision Based" approaches. The Data-Glove based methods use sensor devices for digitizing hand and finger motions into multi-parametric data. The extra sensors make it easy to collect hand configuration and movement. However, these devices are quite expensive and bring much cumbersome experience to the users. In contrast, the Vision Based methods require only a camera, thus realizing a natural interaction between humans and computers without the use of any extra devices.

Proceedings of the $3^{\text {rd }}$ National Conference on Image Processing, Computing, Communication, Networking and Data Analytics (NCICCNDA 2018) 


\section{EXISTING SYSTEM}

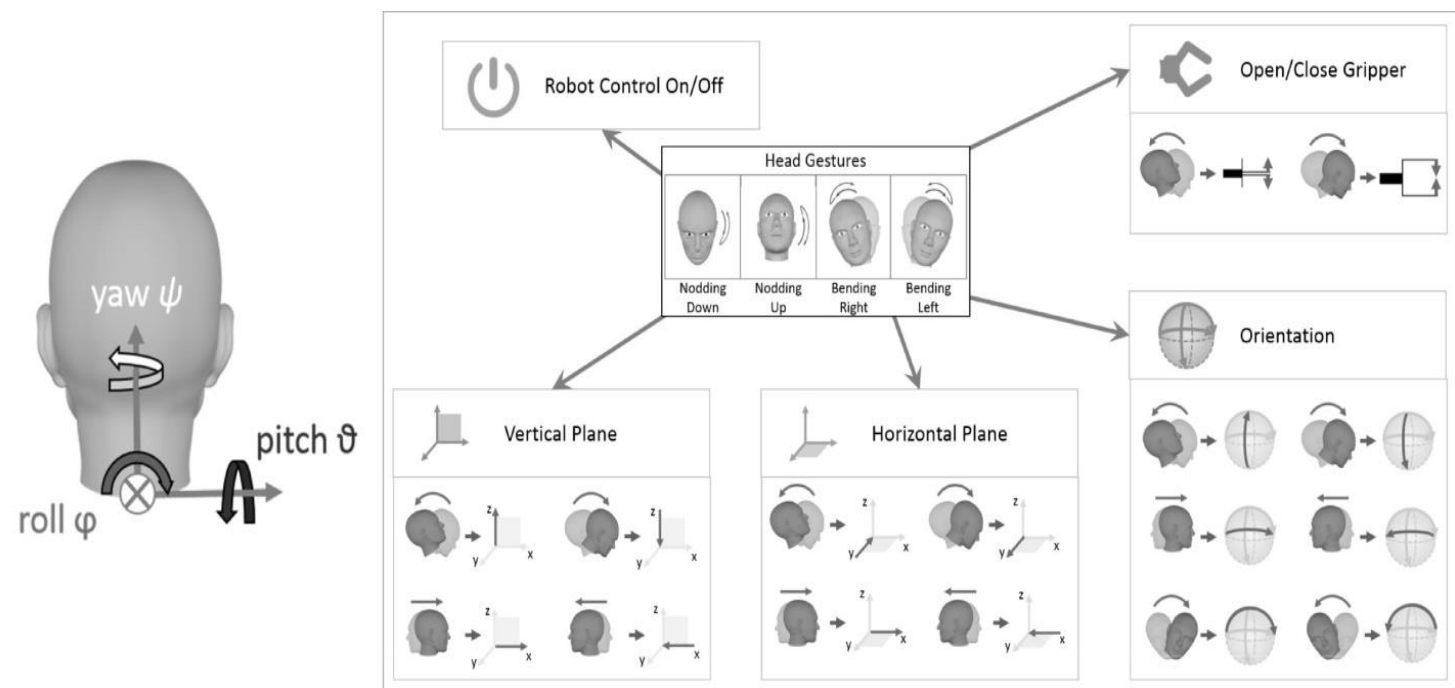

Fig. 1. Interaction design. The human head has three degrees of freedom roll ', pitch \# and yaw. For head motion based control, robot and gripper movements are divided into four control groups. Four head gestures are used to switch between the groups and turn robot control on or off.

The use of glove-based devices has been widely studied in the past for measuring the location and shape of a user's hand. Finger joints angles are measured by some sort of sensor, typically mechanical or optical and the hand position is determined by an additional sensor [5]. Glovebased devices can measure hand postures and locations with high accuracy and high speed. But the use of glove-based devices is not suitable for human computer interfaces because use of these devices restricts the user in his normal way of interacting with computers due to physical connection to the controllers and it doesn't make sense in practical usage.

\section{PROPOSED SYSTEM}

The system mainly includes camera, DB9 connector, speaker \& display. The camera is mainly used to capture the gesture. DB9 connector is used to interface controller with PC. Speaker is used to speak to out gesture input, given by paralyzed person. Based on the gesture using color bands by the paralyzed person. At the output side speaker will Audio output and the message and also LCD displays the message. Paralyzed person also can switch the devices by gestures.

\section{Detection and Tracking:}

We proposed a real time hand tracking technique by using a with red/green color tape placed at fingertip. The proposed approach involves placing red/green colored tape on the hand fingertip. In order to detect the color, a color segmentation method is implemented using the Red Green (RG) color space. We use the intensity based position marking method in order to detect the red color. 


\section{System Architecture}
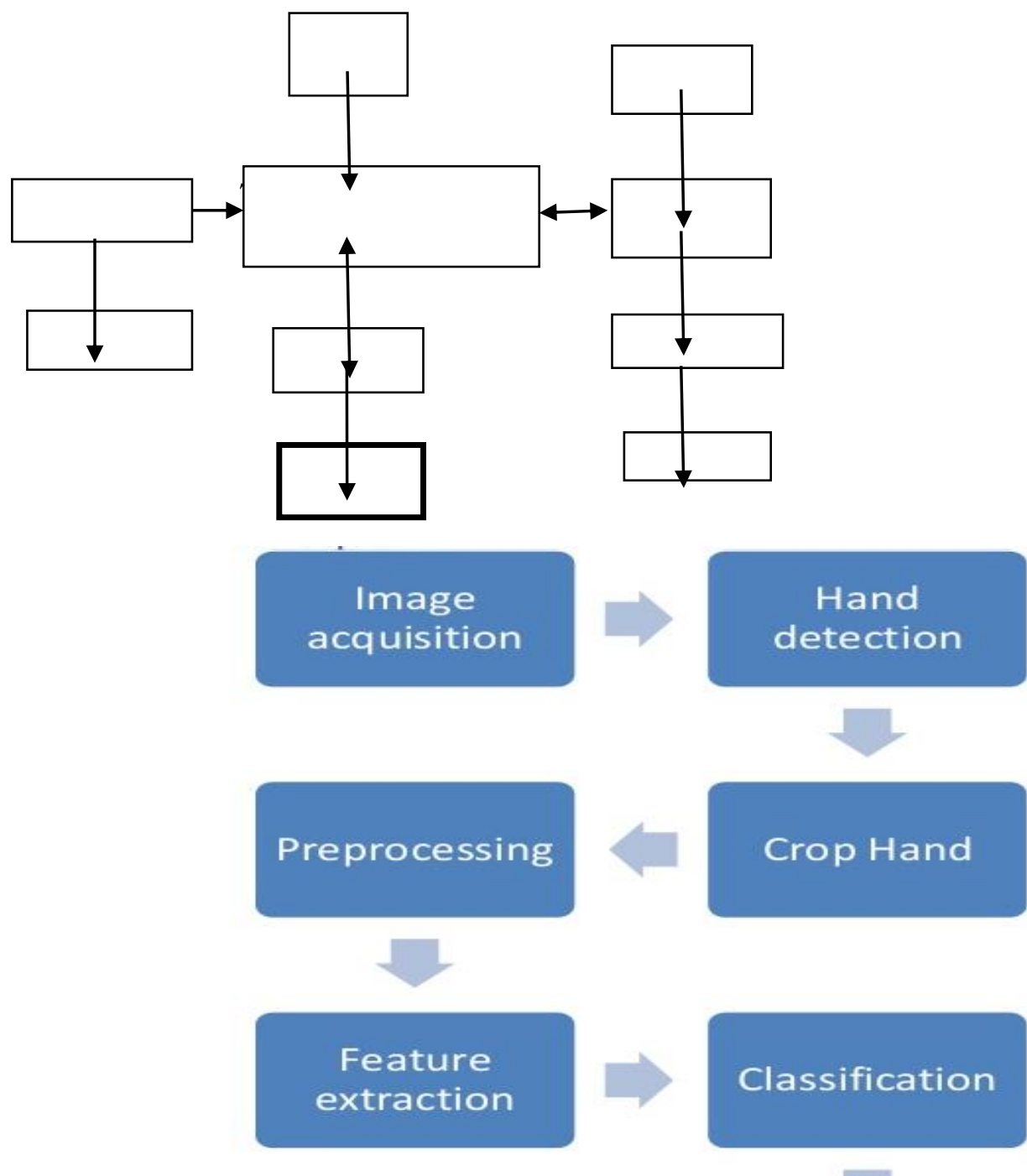

Figure:- Flow chart of proposed system

\section{Methodology}

\section{Algorithm for Color Detection}

Step1: Start

Step2: Capture the video frames using the video input function.

Step2.1: Set the properties of the video object.

Step2.2: Start the video acquisition here.

Proceedings of the $3^{\text {rd }}$ National Conference on Image Processing, Computing, Communication, Networking and Data Analytics (NCICCNDA 2018) 
Gesture based and Voice Enabled Device Switching of Basic Needs for Paralyzed Persons

Step2.3: Set a loop that stop after frames of acquisition.

Step2.3: Get the snapshot of the 2000 current frame.

Step3: Track color blister image subtract the component from gray scale image.

Step3.1: Track RED objects in real time we have to subtract the RED component from the gray scale image to extract the red components in the image.

Step3.2: Track GREEN objects in real time we have to subtract the GREEN component from the gray scale image to extract the green components in the image.

Step4: Apply median filter to remove noise.

Step5: Convert the grayscale image into a binary image (Black and white).

Step6: Remove all those pixels less than 300px.

Step7: Select all the connected components in the image.

Step8: Find Centroid, Area, and Bounding Box using regionprops.

Step8.1: Apply rectangular box for each.

Step9: Stop.

\section{CONCLUSIONS}

In this paper, we proposed a gesture based Device switching and emergency commands generation using image processing (MATLAB) for physically challenged persons and electrical devices (Household devices) like bulb and fan is controlled using Red, Green color bands mounted on fingertip different types of Gestures made and the basic needs of food, water, Medicine and some other basic needs are designed by the FN16-MP voice circuit with the help of SD card Inserted on it. This microcontroller is capable of communicating with transmitter and receiver modules. Presence of every module has been reasoned out and placed carefully thus contributing to the best working of the unit.

\section{References}

[1] International Journal of Advanced Technology in Engineering and Science Volume No.02, IssueNo.05, May2014

[2] http://research.ijcaonline.org/volume79/number13/pxc3891906.pdf

[3] http://ijsetr.org/wp-content/uploads/2013/07/IJSETR-VOL-2-ISSUE-3-749-755.pdf

[4] http://researchtrend.net/ijet41/3\%20DIKSHA\%20NGF.pdf

[5] International Journal of Computer Applications (0975 - 8887) Volume 79 - No 13,

October 2013. 